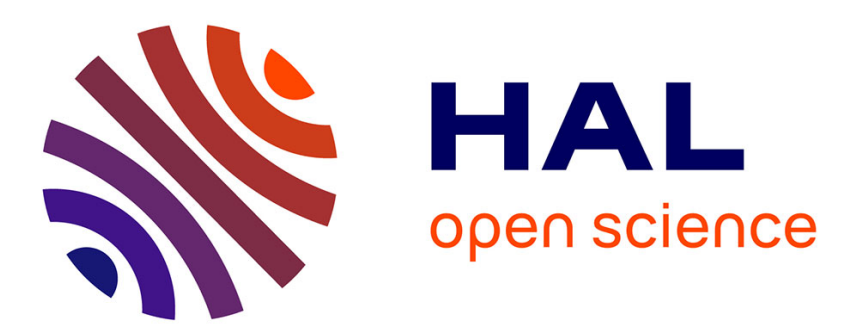

\title{
Revised structure of anthelvencin A and characterization of the anthelvencin biosynthetic gene cluster
}

Céline Aubry, Paolo Clerici, Claude Gerbaud, Laurent Micouin, Jean-Luc

Pernodet, Sylvie Lautru

\section{- To cite this version:}

Céline Aubry, Paolo Clerici, Claude Gerbaud, Laurent Micouin, Jean-Luc Pernodet, et al.. Revised structure of anthelvencin A and characterization of the anthelvencin biosynthetic gene cluster. ACS chemical biology, 2020, 10.1021/acschembio.9b00960 . hal-02502312

\section{HAL Id: hal-02502312 \\ https://hal.science/hal-02502312}

Submitted on 10 Nov 2020

HAL is a multi-disciplinary open access archive for the deposit and dissemination of scientific research documents, whether they are published or not. The documents may come from teaching and research institutions in France or abroad, or from public or private research centers.
L'archive ouverte pluridisciplinaire HAL, est destinée au dépôt et à la diffusion de documents scientifiques de niveau recherche, publiés ou non, émanant des établissements d'enseignement et de recherche français ou étrangers, des laboratoires publics ou privés. 


\section{Revised structure of anthelvencin A and characterization}

\section{2 of the anthelvencin biosynthetic gene cluster}

6 Céline Aubry $^{\mathrm{a}, \mathrm{b}}$, Paolo Clericic ${ }^{\mathrm{c}}$, Claude Gerbaud ${ }^{\mathrm{a}}$, Laurent Micouin ${ }^{\mathrm{c}}$, Jean-Luc

7 Pernodet $^{\mathrm{a}}$, and Sylvie Lautru ${ }^{\mathrm{a}^{*}}$

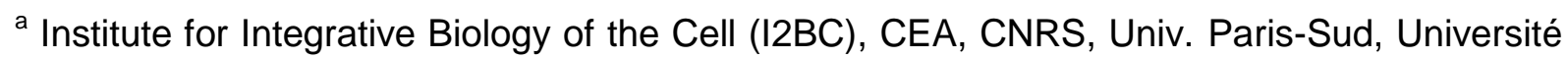

Paris-Saclay, 91198, Gif-sur-Yvette cedex, France

${ }^{\mathrm{b}}$ present address: Unité Molécules de Communication et Adaptation des Microorganismes (MCAM, UMR 7245), Muséum National d'Histoire Naturelle (MNHN), Centre National de la Recherche Scientifique (CNRS), CP 5457 rue Cuvier, 75005 Paris, France

c Université de Paris, Laboratoire de Chimie et Biochimie pharmacologiques et toxicologiques, LCBPT, UMR 8601 CNRS, F-75006 Paris, France 


\section{ABSTRACT}

Anthelvencins A and B are pyrrolamide metabolites produced by Streptomyces venezuelae ATCC 14583 and 14585. Isolated in 1965, they were reported to exhibit anthelmintic and moderate antibacterial activities. In this study, we revise the structure of anthelvencin $A$ and identify a third anthelvencin metabolite, bearing two $\mathrm{N}$-methylated pyrrole groups, which we named anthelvencin C. We sequenced the genome of $S$. venezuelae ATCC 14583 and identified a gene cluster predicted to direct the biosynthesis of anthelvencins. Functional analysis of this gene cluster confirmed its involvement in anthelvencin biosynthesis and allowed us to propose a biosynthetic pathway for anthelvencins. In addition to a nonribosomal peptide synthetase (NRPS), the assembly of anthelvencins involves an enzyme from the ATP-grasp ligase family, Ant23. We propose that Ant23 uses a PCP-loaded 4aminopyrrole-2-carboxylate as substrate. As observed for the biosynthesis of the other pyrrolamides congocidine (produced by Streptomyces ambofaciens ATCC 25877) and distamycin (produced by Streptomyces netropsis DSM 40846), the NRPS assembling anthelvencins is composed of stand-alone domains only. Such NRPSs, sometimes called type II NRPSs, are less studied than the classical multimodular NRPSs. Yet, they constitute an interesting model to study protein-protein interactions in NRPSs and are good candidates for combinatorial biosynthesis approaches. 
Anthelvencins $A$ and $B$ (Figure $1 \mathrm{~A}$ ) are specialized metabolites that were isolated in 1965 from cultures of Streptomyces venezuelae ATCC 14583 and 14585 and exhibit moderate antibacterial and anthelmintic activities ${ }^{1}$. They belong to the family of pyrrolamide metabolites, the best-characterized members of which are congocidine (also called netropsin, produced by Streptomyces ambofaciens ATCC 25877) and distamycin (produced by Streptomyces netropsis DSM 40846) (see Supplementary Figure S1). These metabolites are known to bind to the DNA minor groove with some sequence specificity for regions of four (or more) A or $\mathrm{T}$ bases $^{2}$. During the last decade, the biosynthetic gene clusters of congocidine and distamycin have been identified and the biosynthesis of these metabolites has been elucidated ${ }^{3-7}$. One remarkable aspect of this biosynthesis is that it involves noncanonical non-ribosomal peptide synthetases (NRPSs), solely constituted of stand-alone modules or domains. Such NRPSs are sometimes called type II NRPSs. They participate in the biosynthesis of unusual precursors of non-ribosomal peptides (NRP) ${ }^{8}$ and are involved in the assembly of some NRP such as phosphinothricin ${ }^{9}$ and rhabdopeptide/xenortide peptides $^{10}$.

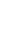

3
two precursors with congocidine and distamycin: 4-acetamidopyrrole-2-carboxylate (5) and 3-aminopropionamidine (6). The remaining precursor is probably 5-amino-3,4-dihydro-2Hpyrrole-2-carboxylate (4), a precursor shared with other pyrrolamides such as kikumycins ${ }^{11}$ or TAN 868A ${ }^{12}$ (Figure S1). In fact, a limited number of precursors seems to be assembled in various combinations to give birth to the different members of the pyrrolamide family. Understanding how these precursors are assembled and combined may improve our comprehension of type II NRPS enzymatic mechanisms and help to design functional synthetic NRPSs using synthetic biology. For these reasons, we undertook to identify and characterize the anthelvencin biosynthetic gene cluster of S. venezuelae ATCC 14583. In this study, we revise the structure of anthelvencin A based on HR-MS ${ }^{2}$ and NMR data. We show that S. venezuelae ATCC 14583 produces, in addition to the already known 
anthelvencins $\mathrm{A}$ and $\mathrm{B}$, a third anthelvencin, methylated on the two pyrrole groups. We named this new anthelvencin anthelvencin C. We also identify the gene cluster directing the biosynthesis of anthelvencins in $S$. venezuelae ATCC 14583 genome and we functionally characterize this gene cluster.

A

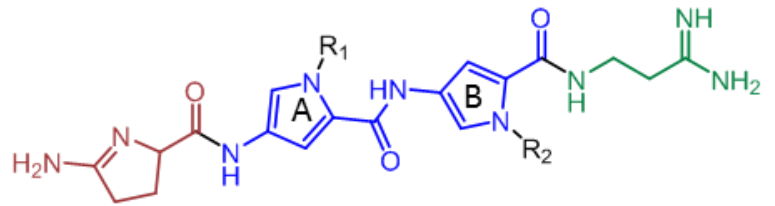

Anthelvencin A original structure (1a) $R_{1}=H, R_{2}=M e$ Anthelvencin $A$ revised structure (1b) $R_{1}=M e, R_{2}=H$ Anthelvencin B (2): $\mathrm{R}_{1}=\mathrm{R}_{2}=\mathrm{H}$ Anthelvencin $\mathrm{C}$ (3): $\mathrm{R}_{1}=\mathrm{R}_{2}=\mathrm{CH}_{3}$

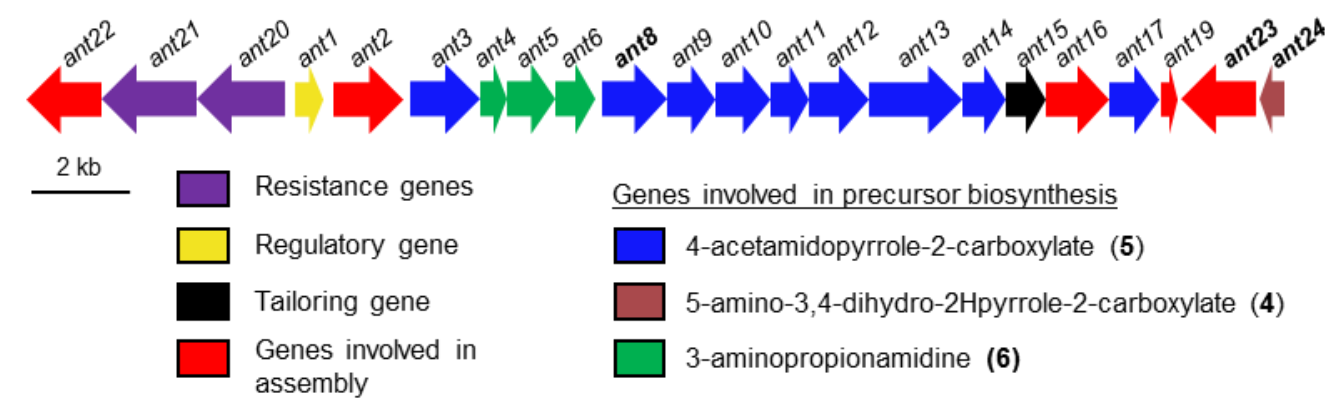

Figure 1. (A) Chemical structure of anthelvencins A, B and C; (B) Genetic organization of the anthelvencin biosynthetic gene cluster in S. venezuelae ATCC 14583. Genes in boldface are genes that were used as targets for genetic inactivation. The numbers in boldface (1a-6) are used to refer to the corresponding chemical structures.

\section{RESULTS AND DISCUSSION}

In silico identification of a gene cluster putatively involved in anthelvencin biosynthesis in S. venezuelae ATCC 14583. To identify the gene cluster directing anthelvencin biosynthesis, we sequenced the genome of the $S$. venezuelae ATCC 14583 strain by the Illumina technology, using a paired-end genomic library. The 5.45 million reads of 301 bps were assembled using Velvet v1.2.10, resulting in 63 contigs with a total length of 9.08 Mbps (180-fold coverage).

The gene cluster directing the biosynthesis of anthelvencins was identified by mining the genome of $S$. venezuelae ATCC 14583 for homologs of genes involved in the biosynthesis of congocidine ${ }^{3}$. We identified a gene cluster (ant, GenBank accession number MK483114) that spans $26 \mathrm{~kb}$ and contains 22 genes (Figure 1B). The deduced products of 
96 twenty of these genes exhibit a high amino acid sequence identity with Cgc proteins involved

97 in congocidine biosynthesis (from 64 to $84 \%$ sequence identity, Table 1 ) and they most likely 98 have

similar 
Table 1. Sequence identities between Ant and Cgc proteins

\begin{tabular}{|c|c|c|c|}
\hline Protein & Putative protein function & $\begin{array}{c}\text { Cgc ortholog } \\
\text { (Accession number) }\end{array}$ & $\begin{array}{c}\% \\
\text { sequence } \\
\text { identity }\end{array}$ \\
\hline Ant1 & Transcriptional regulator & Cgc1 (AKZ59702.1) & 71 \\
\hline Ant2 & NRPS, C domain & Cgc2 (AKZ59703.1) & 66 \\
\hline Ant3 & $\begin{array}{l}\text { 4-Acetamidopyrrole-2-carboxaldehyde } \\
\text { dehydrogenase }\end{array}$ & Cgc3 (AKZ59704.1) & 74 \\
\hline Ant4 & Cytosine monophosphate hydrolase & Cgc4 (AKZ59705.1) & 83 \\
\hline Ant5 & Cytosine reductase & Cgc5 (AKZ59706.1) & 77 \\
\hline Ant6 & Dihydrocytosine hydrolase & Cgc6 (AKZ59707.1) & 78 \\
\hline Ant8 & $\begin{array}{l}\text { Nucleotidyl } \quad \mathrm{N} \text {-acetylglucosamine } \\
\text { dehydrogenase }\end{array}$ & Cgc8 (AKZ59709.1) & 84 \\
\hline Ant9 & $\begin{array}{l}\text { Nucleotidyl-2-acetamido-2- } \\
\text { deoxyglucopyranuronate decarboxylase }\end{array}$ & Cgc9 (AKZ59710.1) & 84 \\
\hline Ant10 & Glycosyltransferase-like enzyme & Cgc10 (AKZ59711.1) & 81 \\
\hline Ant11 & $\begin{array}{l}\mathrm{N} \text {-acetylglucosamine-1-phosphate } \\
\text { nucleotidyltransferase }\end{array}$ & Cgc11 (AKZ59712.1) & 76 \\
\hline Ant12 & $\begin{array}{l}\text { Nucleotidyl threo-2-acetamido-2-deoxy- } \\
\text { pentopyran-4-ulose aminotransferase }\end{array}$ & Cgc12 (AKZ59713.1) & 79 \\
\hline Ant13 & Glycoside hydrolase & Cgc13 (AKZ59714.1) & 78 \\
\hline Ant14 & $\begin{array}{l}\text { 4-Acetamidopyrrole-2-carboxylate } \\
\text { deacetylase }\end{array}$ & Cgc14 (AKZ59715.1) & 80 \\
\hline Ant15 & Methyltransferase & Cgc15 (AKZ59716.1) & 84 \\
\hline Ant16 & NRPS, C domain & Cgc16 (AKZ59717.1) & 68 \\
\hline Ant17 & $\begin{array}{l}\text { 4-Acetamidopyrrole-2-carboxaldehyde } \\
\text { dehydrogenase }\end{array}$ & Cgc17 (AKZ59718.1) & 83 \\
\hline Ant19 & NRPS, PCP domain & Cgc19 (AKZ59720.1) & 64 \\
\hline Ant20 & ABC transporter & Cgc20 (AKZ59701.1) & 81 \\
\hline Ant21 & $A B C$ transporter & Cgc21 (AKZ59700.1) & 81 \\
\hline Ant22 & Acyl co-A synthetase & Cgc22 (AKZ59699.1) & 72 \\
\hline Ant23 & ATP-grasp domain-containing protein & None & \\
\hline Ant24 & Ectoine synthase-like protein & None & \\
\hline
\end{tabular}


function to their Cgc homologs. Thus, the gene numbers attributed to the ant genes were chosen to follow the $c g c$ nomenclature whenever possible. The genetic organization of the ant cluster is remarkably similar to the one of the $c g c$ cluster $^{3}$ from $S$. ambofaciens ATCC23877 (Figure S2). Two cgc genes ( $c g c 7$ and $\operatorname{cgc} 18$ ) have no homologs in the ant gene cluster. These two genes are involved in the biosynthesis of the guanidinoacetate precursor (absent in anthelvencins) and its assembly. Based on the high level of sequence identity between Ant and Cgc proteins and on the previously published biosynthetic pathways for congocidine and distamycin ${ }^{3,4,6,7}$, we propose the following roles for the Ant proteins. Ant8 to Ant13, Ant3 and Ant17 are involved in the biosynthesis of 4acetamidopyrrole-2-carboxylate (5) and Ant14 is involved in its deacetylation. Ant4 to Ant6 are involved in the biosynthesis of 3-aminopropionamidine (6). Ant2, Ant16, Ant19, and Ant22 are involved in the assembly of anthelvencins. In addition to these, proteins are necessary for the biosynthesis and assembly of 5-amino-3,4-dihydro-2H-pyrrole-2carboxylate (4). The ant cluster contains two genes, ant24 and ant23, which have no homolog in the $c g c$ cluster. We hypothesized that they might be involved in the biosynthesis of (4) and its assembly. Indeed, a protein blas ${ }^{13}$ and a conserved domain searches ${ }^{14}$ on the Ant24 sequence suggested that Ant24 belongs to the L-ectoine synthase (EC 4.2.1.108) family of enzymes. L-ectoine synthases catalyze the ring closure of $\mathrm{N} \gamma$-acetyl-L-2,4diaminobutyric acid, yielding the osmolyte ectoine, a metabolite structurally related to (4). In 2011, Witt and collaborators reported that the ectoine synthase from Halomonas elongata can catalyze the intramolecular condensation of glutamine to form (4) as a side reaction ${ }^{15}$. This enzyme has $34 \%$ sequence identity and $51 \%$ sequence similarity with Ant24. Thus, it appears likely that Ant24 catalyzes the same reaction (Scheme 1).

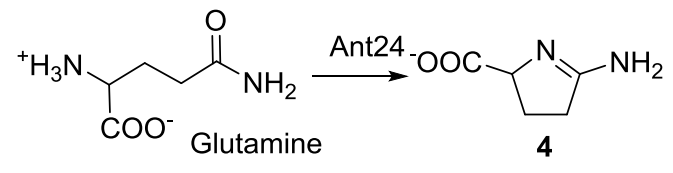


Scheme 1: Proposed biosynthesis of 5-amino-3,4-dihydro-2H-pyrrole-2-carboxylate (4) by Ant24

Ant23 contains an ATP-grasp domain. ATP-grasp enzymes usually catalyze the ATPdependent ligation of a carboxylate-containing molecule to an amino or thiol group-containing molecule $^{16}$. Some of these ATP-grasp enzymes play a role in the biosynthesis of specialized metabolites $^{17}$. They can function as an alternative to or in combination with non-ribosomal peptide synthetases (NRPS), to elongate a peptide chain ${ }^{17,18}$. Thus, it appears plausible that Ant23 catalyzes the amide bond formation between (4) and a PCP (Ant19)-bound 4aminopyrrole-2-carboxylate.

Abolition of the production of four metabolites in a S. venezuelae ATCC 14583 mutant deleted for ant8 (coding for a putative nucleotidyl $\mathbf{N}$-acetylglucosamine dehydrogenase). To verify that the ant gene cluster is involved in the biosynthesis of anthelvencins, we inactivated ant8. This gene is the ortholog of $c g c 8$, the product of which is involved in the biosynthesis of the 4-acetamidopyrrole-2-carboxylate (5). The ant8 gene was replaced by an aac(3)IV resistance cassette by homologous recombination using the pANT007 suicide plasmid, yielding the $S$. venezuelae ANT007 strain. This strain and the wild type S. venezuelae strain were cultivated for three days in MP5 liquid medium. The culture supernatants were then filtered and analyzed by HPLC. The chromatograms show that four metabolites present in the wild type strain supernatant (peaks I to IV) are absent in the supernatant of the ANT007 mutant strain (Figure 2). The first metabolite (peak I, retention time of $11.5 \mathrm{~min}$ ) corresponds to 4-aminopyrrole-2-carboxylate (5), identified by its UV spectrum and by comparison with an authentic standard (Figure 2 and reference 7). The three peaks II (retention time of $13.3 \mathrm{~min}$ ), III (retention time of $14.3 \mathrm{~min}$ ) and IV (retention time of $15.5 \mathrm{~min}$ ) have UV absorption spectra typical of pyrrolamides (Figure S3 and reference 4). 


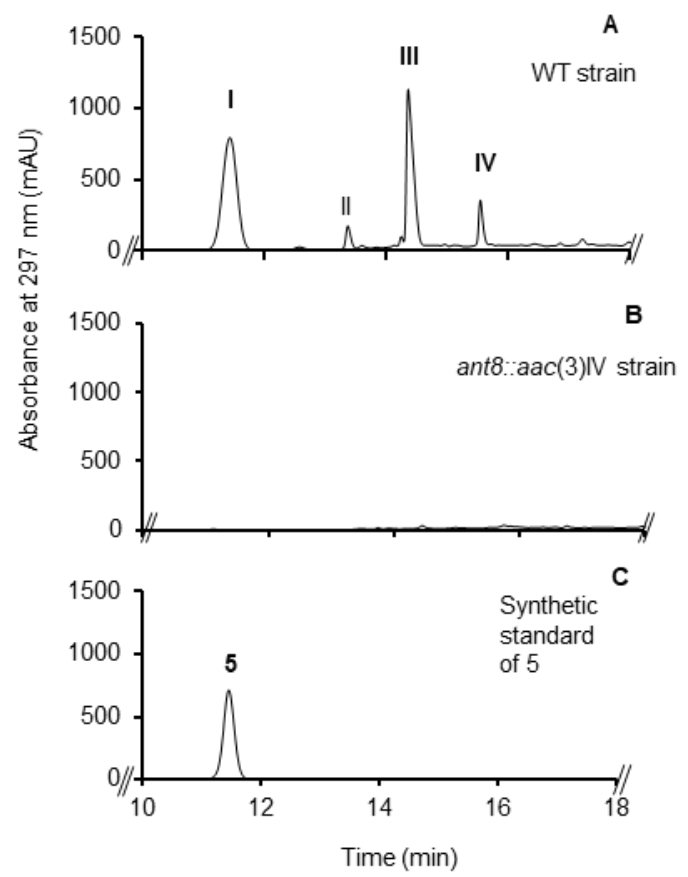

152

153

154

155

156

157

158

159

160

161

162

163

164

165

166

167

168

169

Figure 2: HPLC analysis of culture supernatants of A) S. venezuelae ATCC 14583 wild type and B) ANT007 (S. venezuelae ATCC 14583 ant8::aac(3)IV). C) Standard of 4acetamidopyrrole-2-carboxylate (5). Peaks I to IV are compounds the biosynthesis of which is linked to the ant gene cluster. Peak I corresponds to (5).

Chemical nature of metabolites II, III and IV. To determine the chemical nature of the metabolites II, III and IV, we partially purified them. For that purpose, we used ANT012, a strain that expressed a second copy of the genes ant23 and ant24 under the control of the promoter $\operatorname{rpsL}(\mathrm{TP})^{19}$, as this strain produces compounds III and IV in slightly higher titers (data not shown). The ANT012 culture supernatant was recovered after three days of culture in MP5 medium and the compounds of interest were partially purified on a XAD16 resin. The elution fraction was concentrated to dryness solution, resuspended in water and analyzed by LC-HR-MS ${ }^{2}$.

The exact mass and fragmentation pattern of compound II (Figure S4) are consistent with II being anthelvencin B (2) $\left([\mathrm{M}+\mathrm{H}]^{+} \mathrm{m} / \mathrm{z}=414.1998\right.$; calculated 414.1997). The exact 
mass of compound III (Figure S5) is consistent with III being anthelvencin $A\left([M+H]^{+} \mathrm{m} / \mathrm{z}=\right.$ 428.2151; calculated 428.2153). The fragmentation pattern however (Figure S5, fragments 4 and 5), indicates that the position of the methyl group is not on the B pyrrole ring, as previously proposed (but never experimentally established ${ }^{1}$ ) but rather on the A pyrrole ring (Figure 1). To confirm the structure of anthelvencin A, we purified compound III and carried out NMR experiments. ${ }^{1} \mathrm{H}$ NMR of anthelvencin A hydrochloride proved to be very similar to the one reported for the authentic sample synthesized by Lee and coworkers ${ }^{17}$ assuming the position of the methyl group on the B pyrrole ring. However, a combination of twodimensional NMR experiments confirmed the position of this methyl on the A ring. We therefore propose a revised structure for anthelvencin A (Figure S6).

The exact mass and fragmentation pattern of compound IV (Figure S7) are consistent with IV being an anthelvencin metabolite methylated on both pyrrole groups $\left([\mathrm{M}+\mathrm{H}]^{+} \mathrm{m} / \mathrm{z}=\right.$ 442.2311; calculated 442.2310), a metabolite that we named anthelvencin C (Figure 1A, 3). We tried to purify anthelvencin C to confirm its chemical structure with NMR analyses but this metabolite turned out to be highly unstable, as already observed by Lee and coworkers ${ }^{20}$.

\section{Involvement of ant24 in 5-amino-3,4-dihydro-2H-pyrrole-2-carboxylate (4) biosynthesis.}

To verify that ant24 is involved in the biosynthesis of anthelvencins, we replaced it by an $\operatorname{aac}(3)$ IV resistance cassette by homologous recombination, following the same procedure as described above. The culture supernatant of the resulting mutant strain, called ANT009, was analyzed by HPLC. No production of anthelvencins was observed, confirming that ant24 is necessary for the production of these metabolites (Figure $3 \mathrm{~A}$ ). To ensure that the observed phenotype was due to the replacement of ant24 by the aac(3)IV cassette, we genetically complemented the ANT009 strain using a plasmid expressing ant23 and ant24 under a constitutive promoter. The production of anthelvencins was restored in the complemented 
197 strain, named ANT014 (Figure S8A), thus confirming that ant24 is involved in anthelvencin 198 biosynthesis.

199 To examine Ant24 putative function in the biosynthesis of (4), we undertook the chemical complementation of ANT009 by adding to the culture medium (4), the putative product of Ant24-catalyzed reaction. Thus, (4) was synthetized according to a previously described synthetic procedure ${ }^{21}$ (Scheme 2).

203<smiles>O=C1CCC(C(=O)O)N1</smiles>

Pyroglutamic acid (7)

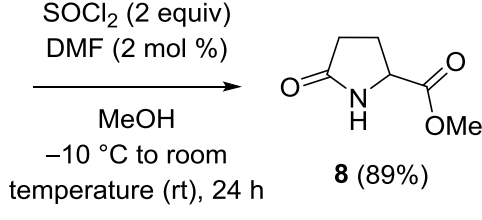

$$
\text { (O) }
$$

4 (quant.)

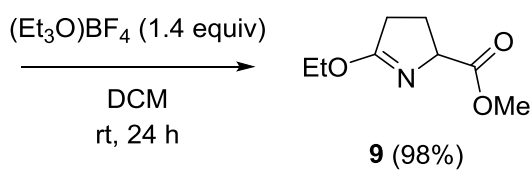

$\mathrm{NaH}_{4} \mathrm{Cl}$ (1.05 equiv) $\mathrm{MeOH}$ reflux, $5 \mathrm{~h}$

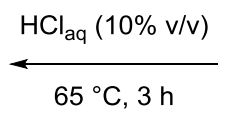

$65^{\circ} \mathrm{C}, 3 \mathrm{~h}$

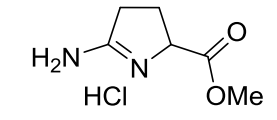

$10(61 \%)$

Scheme 2: Synthesis of 5-amino-3,4-dihydro-2Hpyrrole-2-carboxylate (4)

We next fed the ANT009 strain with (4). As shown in Figure 3B, this resulted in the restoration of the production of anthelvencins $A$ and $C$, hence confirming the involvement of ant24 in the biosynthesis of the anthelvencin precursor (4). 


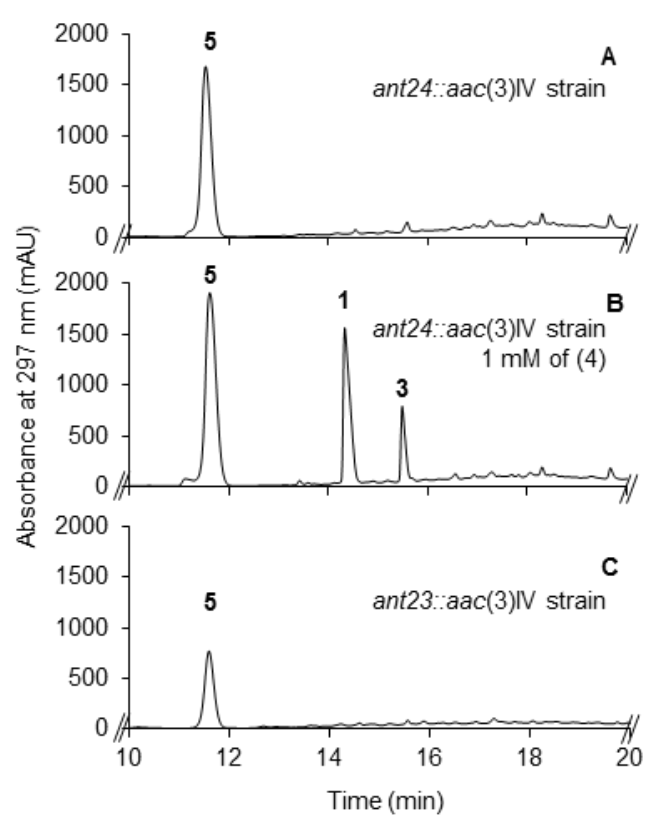

Figure 3: HPLC analysis of culture supernatants of (A) ANT009 (S. venezuelae ATCC 14583 ant24::aac(3)IV), (B) ANT009 (S. venezuelae ATCC 14583 ant24::aac(3)IV) cultivated in presence of $1 \mathrm{mM}$ of (4), and (C) ANT008 (S. venezuelae ATCC 14583 ant23::aac(3)IV). Numbers above peaks correspond to the metabolite numbers in the text.

Involvement of ant23 in the biosynthesis of anthelvencins. To confirm that Ant23 is involved in anthelvencin biosynthesis, we replaced ant23 by the aac(3)IV resistance cassette following the previously described protocol. The resulting mutant strain was called ANT008. It was cultivated for three days in MP5 medium at $28^{\circ} \mathrm{C}$ and the culture supernatant was analyzed by HPLC. Figure $3 \mathrm{C}$ shows that no anthelvencin is produced by the ANT008 mutant. To ensure that the observed phenotype was due to the replacement of ant23 by the aac(3)IV cassette, we genetically complemented the ANT008 strain using the plasmid expressing ant23 and ant24 under a constitutive promoter. The production of anthelvencins was restored in the complemented strain, named ANT013 (Figure S8B), thus confirming that ant23 is involved in anthelvencin biosynthesis.

Proposed biosynthetic pathway for anthelvencin biosynthesis. Based on the results presented above and on previous characterizations of pyrrolamide biosyntheses ${ }^{3,4,6}$, we 
proposed that anthelvencins are assembled from 3-amidinopropionamidine, 4-aminopyrrole2-carboxylate and 5-amino-3,4-dihydro-2H-pyrrole-2-carboxylate following the biosynthetic pathway presented in Figure 4. As already observed for the biosynthesis of other pyrrolamides (congocidine, distamycin), the non-ribosomal peptide synthetase involved in

234 anthelvencins is constituted solely of stand-alone domains ( $\mathrm{C}$ domains Ant2 and Ant16 and 235 PCP domain Ant19). No adenylation domain is involved in the activation of the carboxylate 236 groups of the precursors. Instead, activation of the carboxylate group of the pyrrole precursor 237 (5) and the covalent attachment of the activated precursor to the PCP domain Ant19 is catalyzed by Ant22, which belongs to the family of acyl-CoA synthetases. The formation of 239 the first amide bond between (4) and Ant19-bound (5) is likely catalyzed by Ant23, an 240 enzyme from the ATP-grasp ligase family, which form acylphosphate intermediates. Two 241 stand-alone condensation domains, Ant16 and Ant2, catalyze the formation of the other 242 amide bonds, adding respectively a second pyrrole precursor and the 3243 aminopropionamidine to Ant19-bound intermediates. Thus, during anthelvencin assembly, 244 the stand-alone PCP domain, Ant19, interacts with two different families of enzymes 245 (condensation domain and ATP-grasp ligase). 

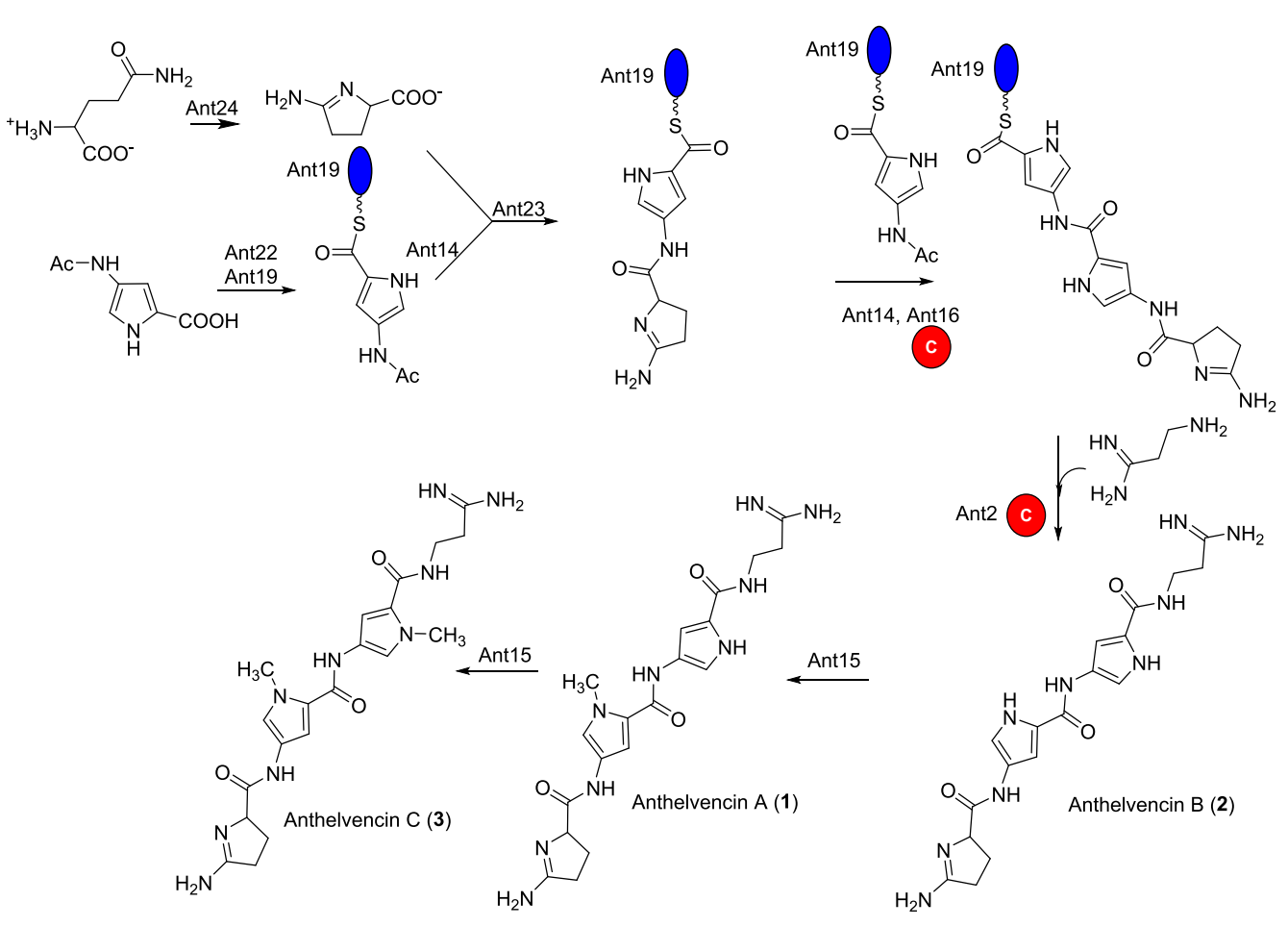

Figure 4: Proposed biosynthetic pathway for anthelvencins $A, B$ and $C$ The blue ellipses represent PCP domains, and the red circles represent $C$ domains

In conclusion, we have identified and characterized the gene cluster directing the biosynthesis of anthelvencins in Streptomyces venezuelae ATCC 14583 . We showed that this cluster directs the biosynthesis of two known metabolites, anthelvencin A, for which we propose a revised structure, anthelvencin $B$, and new anthelvencin that we named anthelvencin C. As already observed for the assembly of other pyrrolamides (congocidine, distamycin), the assembly of anthelvencins involves a non-canonical NRPS constituted of stand-alone domains only. Such structural organization, rarely encountered in NRPS, appears to be characteristic of pyrrolamide NRPSs. Characterizing biosynthetic pathways involving type II NRPSs is of interest for the production of novel specialized metabolites by synthetic biology as the architecture of these NRPSs is particularly suited for combinatorial biosynthesis approaches. 
Bacterial strains, plasmids and growth conditions. Strains and plasmids used in this study are listed in Table S1 and S2. Escherichia coli strains were grown at $37^{\circ} \mathrm{C}$ in LB or $\mathrm{SOB}$ complemented with $\mathrm{MgSO}_{4}(20 \mathrm{mM}$ final), supplemented with appropriate antibiotics as needed $^{22}$. The Soya Flour Mannitol (SFM) medium ${ }^{23}$ was used for genetic manipulations of Streptomyces strains and spore stocks preparations at $28^{\circ} \mathrm{C}$. Streptomyces strains were grown for three days at $28^{\circ} \mathrm{C}$ in $M P 5^{24}$ for anthelvencins (1-3) production.

DNA preparation and manipulations. All oligonucleotides used in this study were purchased from Eurofins and are listed in Table S3. The High fidelity DNA polymerase Phusion (Thermo Fisher Scientific) was used to amplify the DNA fragments for the construction of the suicide plasmids. DreamTaq polymerase (Thermo Fisher Scientific) was used for PCR verification of plasmids and of the replacement of the targeted genes by the resistance cassette aac(3)IV. DNA fragments were purified from agarose gels using the Nucleospin Gel and PCR clean-up kit from Macherey-Nagel. E. coli transformations and $E$. coli/Streptomyces conjugations were performed according to standard procedures ${ }^{25,23}$.

S. venezuelae ATCC 14583 sequencing and assembly. Total DNA of S. venezuelae ATCC 14583 was extracted following the salting out procedure ${ }^{23}$ except that the mycelium was ground in liquid nitrogen. A paired-end library of the whole genome was constructed and sequenced at the high throughput sequencing core facility of I2BC with a MiSeq M01342 instrument (Illumina), generating 5.45 million 301 bp reads that were assembled using Velvet v1.2.10. The GenBank accession number of the anthelvencin gene cluster is MK483114.

Construction of the replacement mutants. The suicide plasmid pANT007 was constructed to replace the ant8 gene by an aac(3)IV resistance cassette in $S$. venezuelae. This vector was constructed by assembling the three following inserts in the backbone pOSV400 (bearing a hygromycin resistance gene) ${ }^{26}$ : a $1.8 \mathrm{~kb}$ fragment upstream of ant8, the resistance cassette $\operatorname{aac}(3) \mathrm{IV}$ and a $2.0 \mathrm{~kb}$ DNA fragment downstream of ant8. The $1.8 \mathrm{~kb}$ and $2.0 \mathrm{~kb}$ 
DNA fragments from S. venezuelae ATCC 14583 were amplified by PCR with the primers CEA001/CEA002 and CEA003/CEA004 respectively. The PCR products were purified and ligated into $\mathrm{pCR} \circledast$ Blunt, yielding pANT001 (containing the upstream DNA fragment) and pANT002 (containing the downstream DNA fragment). Both plasmids were verified by sequencing (Genewiz). The $\operatorname{aac}(3) \mathrm{IV}$ resistance cassette was obtained by digestion of pW60 $0^{27}$ by HindllI. The $1.8 \mathrm{~kb}$ Hindll-Xhol fragment from pANT001, the $1.0 \mathrm{~kb}$ HindllI aac(3)IV fragment, and the $2.0 \mathrm{~kb}$ Hindlll-Spel fragment from pANT002 were then ligated into the Xhol-Spel-digested pOSV400, yielding pANT007. The pANT007 plasmid was verified by restriction digestion and was introduced into S. venezuelae ATCC 14583 by intergeneric conjugation from the E. coli ET12567/pUZ8002/pANT007 strain. Doublerecombinant mutants were selected on SFM plates with $50 \mu \mathrm{g} / \mathrm{mL}$ apramycin and screened for hygromycin sensitivity. DNA from the resulting strain, ANT007, was isolated and analyzed by PCR using the primers A5, A6, and CEA013-CEA016 (See Figure S8). The same protocol was used for the construction of the ANT008 mutant strain (replacement of ant23), using the plasmid pANT008, and the ANT009 mutant strain (replacement of ant24), using the plasmid pANT009 (see Tables S2 and S3 for plasmid names and for primer sequences).

Construction of the ANT012 strain overexpressing Ant23 and Ant24. The DNA region containing ant23-ant24 was amplified by PCR from S. venezuelae ATCC 14583 genomic DNA using the primers CEA034/CEA035. The PCR product was purified and cloned into pCR® Blunt, yielding pANT011. The sequence of the insert was confirmed by sequencing. The $2.0 \mathrm{~kb}$ Nhel/Afll fragment from pANT011 was ligated into the Spel/Aflll-digested pCEA00 $5^{28}$. The obtained plasmid, containing ant23 and ant24 under the $\operatorname{rpsL}(\mathrm{TP})$ promoter, was named pANT012 and confirmed by restriction digestion. This plasmid was introduced into S. venezuelae ATCC 14583 by intergeneric conjugation. The correct integration of pANT012 was verified on the extracted DNA by PCR using the primers CEA_vec_seq14 and CEA_vec_seq15 and the strain was named ANT012. 
Genetic complementation of ANT008. As the ANT008 strain bears the aac(3)IV resistance marker, the previously constructed pANT012 plasmid, bearing an aac(3)IV resistance gene cassette, could not be used for the genetic complementation of the strain. Thus, the $2.4 \mathrm{~kb}$ Nsil/AfII DNA fragment of pANT012 containing ant23 and ant24 under the control of the $\operatorname{rpsL}(\mathrm{TP})$ promoter was ligated into the Nsil/Afnl-digested pOSV806 ${ }^{28}$. The resulting plasmid was named pANT013 and was introduced into ANT008 by intergeneric conjugation. The strain obtained was named ANT013.

Chemical synthesis of 5-amino-3,4-dihydro-2H-pyrrole-2-carboxylate (4). Compound (4) was prepared according to a previously described synthetic procedure ${ }^{29}$ (Scheme 2). Commercially available DL-pyroglutamic acid (7) was converted into the corresponding methyl ester (8) by treatment with thionyl chloride (2 equiv.), and N,N-dimethylformamide (DMF) (2 mol \%) in methanol. Derivative (8) was then submitted to a reaction with triethyloxonium tetrafluoroborate (Meerwein's salt, 1.4 equiv.) in dichloromethane (DCM) to form carboximidate (9) in quantitative yield. This compound subsequently reacted with ammonium chloride (1.05 equiv.) in refluxing methanol to provide product (10) in $61 \%$ yield. Hydrolysis of the ester moiety of compound (10) finally afforded the desired acid (4) in a quantitative yield. A detailed synthesis protocol is available in the Supporting Information.

Chemical complementation of ANT009. S. venezuelae ANT009 strain was cultivated in $50 \mathrm{~mL}$ of MP5. After $24 \mathrm{~h}$, the cultures were separated in two $25 \mathrm{~mL}$ cultures, and $1 \mathrm{mM}$ of (4) (final concentration) was added to one of the cultures. After a total of $72 \mathrm{~h}$ of cultivation, culture supernatants were analyzed by HPLC as described below.

HPLC analysis of culture supernatants. S. venezuelae ATCC 14583 and its derivatives were cultivated in MP5 medium for three days at $28^{\circ} \mathrm{C}$. The supernatants were filtered using Mini-UniPrep syringeless filter devices (0.2 $\mu \mathrm{m}$, Whatman). The samples were analyzed on an Atlantis $\mathrm{C}_{18}$ T3 column ( $250 \mathrm{~mm} \times 4.6 \mathrm{~mm}, 5 \mu \mathrm{m}$, column temperature $28^{\circ} \mathrm{C}$ ) using an 
Agilent 1200 HPLC instrument with a quaternary pump. Samples were eluted in isocratic conditions with $0.1 \% \mathrm{v} / \mathrm{v} \mathrm{HCOOH}$ in $\mathrm{H}_{2} \mathrm{O}$ (solvent $\mathrm{A}$ )/ $0.1 \% \mathrm{v} / \mathrm{v} \mathrm{HCOOH}$ in $\mathrm{CH}_{3} \mathrm{CN}$ (solvent $\mathrm{B}$ ) $(95: 5)$ at $1 \mathrm{ml} \cdot \mathrm{min}^{-1}$ for $7 \mathrm{~min}$, followed by a gradient to 40:60 A/B over $23 \mathrm{~min}$. Anthelvencins were detected by monitoring absorbance at $297 \mathrm{~nm}$.

LC-HR-MS-MS analyses. The resuspended elution fraction obtained above was analyzed by LC-HR-MS ${ }^{2}$. The analysis was performed using a Dionex Ultimate 3000 HPLC system coupled with a Maxis IIM QTOF mass spectrometer (Bruker, MA, USA) fitted with an electrospray ionization (ESI) source.

Chromatographic analysis was performed using a $\mathrm{C}_{18}$ AcclaimTM RSLC PolarAdvantage II (2.1 x $100 \mathrm{~mm}, 2.2 \mu \mathrm{m}$ pore size) column (Thermo Scientific, MA, USA). Column temperature was set at $40{ }^{\circ} \mathrm{C}$ and $2 \mu \mathrm{L}$ of each sample was injected via an autosampler cooled to $4{ }^{\circ} \mathrm{C}$. A flow rate of $0.3 \mathrm{~mL} / \mathrm{min}$ was used and the eluent was introduced directly into the MS for ion detection. Elution was conducted with a mobile phase consisting of $0.1 \% \mathrm{v} / \mathrm{v} \mathrm{HCOOH}$ in $\mathrm{H}_{2} \mathrm{O}$ (solvent $\mathrm{A}$ ) and $0.1 \% \mathrm{v} / \mathrm{v} \mathrm{HCOOH}$ in $\mathrm{CH}_{3} \mathrm{CN}$ (solvent B) following the gradient elution profile: $0 \mathrm{~min}, 5 \%$ solvent $\mathrm{B} ; 2 \mathrm{~min}, 5 \%$ solvent $\mathrm{B} ; 9 \mathrm{~min}$, $50 \%$ solvent $B ; 15$ min $90 \%$ solvent $B ; 17$ min $90 \%$ solvent $B ; 19$ min $5 \%$ solvent $B ; 21$ min $5 \%$ solvent B. In the first half minute of each run, a sodium formate solution was injected directly as an internal reference for calibration. The acquisition parameters of the ESI source were set up as follows: electrospray voltage for the ESI source: $3500 \mathrm{~V}$, nebulising gas (N2) pressure: 35 psi, drying gas $\left(\mathrm{N}_{2}\right)$ flow: $8 \mathrm{~L} / \mathrm{min}$, and drying temperature: $200^{\circ} \mathrm{C}$. Mass spectra were recorded over the $\mathrm{m} / \mathrm{z}$ range $100-1300$ at a frequency of $2 \mathrm{~Hz}$, in positive ion mode. For MS/MS analysis, the cycle time was of $3 \mathrm{sec}$. Mass spectra were recorded over the $\mathrm{m} / \mathrm{z}$ range 100-1300 at a frequency of $2 \mathrm{~Hz}$, in positive ion mode. Selected parent ion at $\mathrm{m} / \mathrm{z}$ 442.23 was fragmented at a fixed collision energy value of $40 \mathrm{eV}$ and an isolation window of $0.5 \mathrm{amu}$. 
Accession codes. The GenBank accession number of the anthelvencin gene cluster is MK483114. Its MiBig ${ }^{30}$ accession number is BGC0002042.

Supporting Information Available: The protocol for the synthesis of 5-amino3,4-dihydro-2H-pyrrole-2-carboxylate (4), the strains, plasmids and oligonucleotides used in this study and seven supplementary figures are available in the Supporting Information. This material is available free of charge via the internet at http://pubs.acs.org

\section{Acknowledgements}

We acknowledge the High-throughput sequencing facility of I2BC for its sequencing and bioinformatics expertise (Centre de Recherche de Gif - http://www.i2bc.paris-saclay.fr/). We thank Z. Hong, Y. Li, S. Prado and D. Touboul for their help with the LC-HRMS ${ }^{2}$ analysis. The research received funding from ANR-14-CE16-0003-01. The funders had no role in study design, data collection and interpretation, or the decision to submit the work for publication.

\section{References}

(1) Probst, G. W., Hoehn, M. M., and Woods, B. L. (1965) Anthelvencins, new antibiotics with anthelmintic properties. Antimicrob. Agents Chemother. 5, 789-795.

(2) Neidle, S. (2001) DNA minor-groove recognition by small molecules. Nat. Prod. Rep. 18, 291-309.

(3) Juguet, M., Lautru, S., Francou, F.-X., Nezbedová, S., Leblond, P., Gondry, M., and Pernodet, J.-L. (2009) An iterative nonribosomal peptide synthetase assembles the pyrroleamide antibiotic congocidine in Streptomyces ambofaciens. Chem. Biol. 16, 421-431. (4) Vingadassalon, A., Lorieux, F., Juguet, M., Le Goff, G., Gerbaud, C., Pernodet, J.-L., and Lautru, S. (2015) Natural combinatorial biosynthesis involving two clusters for the synthesis of three pyrrolamides in Streptomyces netropsis. ACS Chem. Biol. 10, 601-610.

(5) Hao, C., Huang, S., Deng, Z., Zhao, C., and Yu, Y. (2014) Mining of the pyrrolamide antibiotics analogs in Streptomyces netropsis reveals the amidohydrolase-dependent "iterative strategy" underlying the pyrrole polymerization. PloS One 9, e99077.

(6) Al-Mestarihi, A. H., Garzan, A., Kim, J. M., and Garneau-Tsodikova, S. (2015) Enzymatic Evidence for a revised congocidine biosynthetic pathway. Chembiochem Eur. J. Chem. Biol. $16,1307-1313$. 
(7) Lautru, S., Song, L., Demange, L., Lombès, T., Galons, H., Challis, G. L., and Pernodet, J.-L. (2012) A sweet origin for the key congocidine precursor 4-acetamidopyrrole-2carboxylate. Angew. Chem. Int. Ed Engl. 51, 7454-7458.

(8) Jaremko, M. J., Davis, T. D., Corpuz, J. C., and Burkart, M. D. (2020) Type II nonribosomal peptide synthetase proteins: structure, mechanism, and protein-protein interactions. Nat. Prod. Rep. https://doi.org/10.1039/C9NP00047J

(9) Schinko, E., Schad, K., Eys, S., Keller, U., and Wohlleben, W. (2009) Phosphinothricintripeptide biosynthesis: an original version of bacterial secondary metabolism?

Phytochemistry 70, 1787-1800.

(10) Cai, X., Nowak, S., Wesche, F., Bischoff, I., Kaiser, M., Fürst, R., and Bode, H. B. (2017) Entomopathogenic bacteria use multiple mechanisms for bioactive peptide library design. Nat. Chem. 9, 379-386.

(11) Takaishi, T., Sugawara, Y., and Suzuki, M. (1972) Structure of Kikumycin A and B. Tetrahedron Lett. 13, 1873-1876.

(12) Takizawa, M., Tsubotani, S., Tanida, S., Harada, S., and Hasegawa, T. (1987) A new pyrrole-amidine antibiotic TAN-868 A. J. Antibiot. (Tokyo) 40, 1220-1230.

(13) Altschul, S. F., Gish, W., Miller, W., Myers, E. W., and Lipman, D. J. (1990) Basic local alignment search tool. J. Mol. Biol. 215, 403-410.

(14) Marchler-Bauer, A., Bo, Y., Han, L., He, J., Lanczycki, C. J., Lu, S., Chitsaz, F., Derbyshire, M. K., Geer, R. C., Gonzales, N. R., Gwadz, M., Hurwitz, D. I., Lu, F., Marchler, G. H., Song, J. S., Thanki, N., Wang, Z., Yamashita, R. A., Zhang, D., Zheng, C., Geer, L. Y., and Bryant, S. H. (2017) CDD/SPARCLE: functional classification of proteins via subfamily domain architectures. Nucleic Acids Res. 45, D200-D203.

(15) Witt, E. M. H. J., Davies, N. W., and Galinski, E. A. (2011) Unexpected property of ectoine synthase and its application for synthesis of the engineered compatible solute ADPC. Appl. Microbiol. Biotechnol. 91, 113-122.

(16) Galperin, M. Y., and Koonin, E. V. (1997) A diverse superfamily of enzymes with ATPdependent carboxylate-amine/thiol ligase activity. Protein Sci. Publ. Protein Soc. 6, 26392643.

(17) Goswami, A., and Van Lanen, S. G. (2015) Enzymatic strategies and biocatalysts for amide bond formation: tricks of the trade outside of the ribosome. Mol. Biosyst. 11, 338-353. (18) Hollenhorst, M. A., Clardy, J., and Walsh, C. T. (2009) The ATP-dependent amide ligases DdaG and DdaF assemble the fumaramoyl-dipeptide scaffold of the dapdiamide antibiotics. Biochemistry 48, 10467-10472.

(19) Shao, Z., Rao, G., Li, C., Abil, Z., Luo, Y., and Zhao, H. (2013) Refactoring the Silent Spectinabilin Gene Cluster Using a Plug-and-Play Scaffold. ACS Synth. Biol. 2, 662-669.

(20) Lee, M., Coulter, D. M., and Lown, J. W. (1988) Total synthesis and absolute configuration of the antibiotic oligopeptide (4S)-(+)-anthelvencin A and its 4R-(-) enantiomer. J. Org. Chem. 53, 1855-1859.

(21) Lee, M., and Lown, J. W. (1987) Synthesis of (4S)- and (4R)-methyl 2-amino-1pyrroline-5-carboxylate and their application to the preparation of (4S)-(+)- and (4R)-(-)dihydrokikumycin B. J. Org. Chem. 52, 5717-5721.

(22) Sambrook, J., and Russell, D. (2000) Molecular Cloning: A Laboratory Manual 3rd Revised edition. Cold Spring Harbor Laboratory Press,U.S.

(23) Kieser, T., Bibb, M., Buttner, M., and Hopwood, D. A. (2000) Practical Streptomyces Genetics, John Innes Foundation, Norwich NR47UH, UK.

(24) Pernodet, J. L., Alegre, M. T., Blondelet-Rouault, M. H., and Guérineau, M. (1993) Resistance to spiramycin in Streptomyces ambofaciens, the producer organism, involves at least two different mechanisms. J. Gen. Microbiol. 139, 1003-1011.

(25) Sambrook, J., and Russell, D. W. (2001) Molecular cloning: A Laboratory Manual, Third edition. CSHL Press, Cold Spring Harbor, NY.

(26) Boubakri, H., Seghezzi, N., Duchateau, M., Gominet, M., Kofroňová, O., Benada, O., Mazodier, P., and Pernodet, J.-L. (2015) The Absence of pupylation (prokaryotic ubiquitinlike protein modification) affects morphological and physiological differentiation in Streptomyces coelicolor. J. Bacteriol. 197, 3388-3399. 
(27) Corre, C., Song, L., O'Rourke, S., Chater, K. F., and Challis, G. L. (2008) 2-Alkyl-4hydroxymethylfuran-3-carboxylic acids, antibiotic production inducers discovered by Streptomyces coelicolor genome mining. Proc. Natl. Acad. Sci. U. S. A. 105, 17510-17515. (28) Aubry, C., Pernodet, J.-L., and Lautru, S. (2019) Modular and Integrative Vectors for Synthetic Biology Applications in Streptomyces spp. Appl. Environ. Microbiol. 85. (29) Lee, M., and Lown, J. W. (1987) Synthesis of (4S)- and (4R)-methyl 2-amino-1pyrroline-5-carboxylate and their application to the preparation of (4S)-(+)- and (4R)-(-)dihydrokikumycin B. J. Org. Chem. 52, 5717-5721. (30) Kautsar, S. A., Blin, K., Shaw, S., Navarro-Muñoz, J. C., Terlouw, B. R., van der Hooft, J. J. J., van Santen, J. A., Tracanna, V., Suarez Duran, H. G., Pascal Andreu, V., SelemMojica, N., Alanjary, M., Robinson, S. L., Lund, G., Epstein, S. C., Sisto, A. C., Charkoudian, L. K., Collemare, J., Linington, R. G., Weber, T., and Medema, M. H. (2020) MIBiG 2.0: a repository for biosynthetic gene clusters of known function. Nucleic Acids Res. 48, D454D458. 